\title{
Minimal Sufficient Conditions for a Primal Optimizer in Nonsmooth Utility Maximization
}

\author{
Nicholas Westray • Harry Zheng.
}

\begin{abstract}
We continue the study of utility maximization in the nonsmooth setting and give a counterexample to a conjecture made in [3] on the optimality of random variables valued in an appropriate subdifferential. We derive minimal sufficient conditions on a random variable for it to be a primal optimizer in the case where the utility function is not strictly concave.
\end{abstract}

Keywords Nonsmooth utility maximization · Convex duality · Subdifferential wealth random variables.

Mathematics Subject Classification (2000) 93E20 - 49J52 · 60H30

JEL Classification G10 - G11

\section{Introduction}

There is a considerable body of literature devoted to the utility maximization problem in both complete and incomplete markets, see Karatzas and Žitković [7] and Kramkov and Schachermayer [8] for an excellent overview. The standard approach first finds a solution $Y_{*}$ to an appropriate dual problem and then uses the conjugacy relations to construct the optimal terminal wealth $X_{*}$. The utility function is usually assumed to be strictly concave and continuously differentiable, it then follows, as shown in [8], that $X_{*}=-\tilde{U}^{\prime}\left(Y_{*}\right)$, where $\tilde{U}$ is the conjugate function to $U$.

More recently, several authors consider the problem without these assumptions on the utility function, see Bouchard [1], Bouchard, Touzi and Zeghal [2] and Deelstra, Pham and Touzi [3]. In these articles the authors show that

This research was funded by an EPSRC grant

N. Westray

Department of Mathematics, Humboldt Universität Berlin, Unter den Linden 6, 10099 Berlin E-mail: westray@math.hu-berlin.de

H. Zheng

Department of Mathematics, Imperial College, South Kensington, London, SW7 2AZ

E-mail: h.zheng@imperial.ac.uk 
there exists a replicable random variable $X_{*}$ valued in $-\partial \tilde{U}\left(Y_{*}\right)$, the subdifferential of $\tilde{U}$, which satisfies a suitable budget constraint with equality and maximizes the expected utility.

When $U$ is not strictly concave the set $\partial \tilde{U}$ is no longer a singleton and a smooth approximation technique (quadratic-inf-convolution) together with convergence analysis have to be used to construct the optimal terminal wealth $X_{*}$, see $[2,3]$ for details. A natural question is then whether any random variable $X_{*}$ chosen in $-\partial \tilde{U}\left(Y_{*}\right)$, satisfying a suitable budget constraint equality, is optimal for the utility maximization problem. If this were true it would simplify greatly the solution procedure, whereas if not then it would imply that the complicated approximation techniques used in $[2,3]$ are essential.

It is conjectured in [3] that it is indeed the case that such random variables are optimal. Our first contribution is to give a simple counterexample to show that this is in fact not true in general. We then go on to a natural generalization of this conjecture and provide necessary and sufficient conditions for a subdifferential valued random variable to be optimal for the primal utility maximization problem when the utility function may not be smooth or strictly concave.

The paper is organized as follows. Section 2 formulates the conjecture by giving the necessary background. Section 3 discusses the counterexample. Section 4 looks at a generalization.

\section{Nonsmooth Utility Maximization and the Conjecture}

The setup is the standard one in mathematical finance. There is a finite time horizon $T$ and a market consisting of one bond, assumed constant, and $d$ stocks, $S^{1}, \ldots, S^{d}$ modelled by an $\mathbb{R}^{d}$-valued semimartingale on a filtered probability space $\left(\Omega, \mathcal{F},\left(\mathcal{F}_{t}\right)_{0 \leq t \leq T}, \mathbb{P}\right)$, satisfying the usual conditions. We write $X$ for the process $\left(X_{t}\right)_{0 \leq t \leq T}$ and "for all $t$ " implicitly meaning "for all $t \in[0, T]$ ". For a predictable $S$-integrable process, we use $H \cdot S$ to denote the stochastic integral with respect to $S$ and refer the reader to Jacod and Shiryaev [6] and Protter [9] for further details.

For our primal maximization problem we are interested in the nonnegative wealth processes

$$
X^{x, H}=x+H \cdot S,
$$

where $x>0$ and $H$ is a predictable $S$-integrable process such that $x+H \cdot S \geq 0$. The set for optimization is now the following,

$$
\mathcal{X}(x):=\left\{X \in L_{+}^{0}(\mathbb{P}): X \leq X_{T}^{x, H} \text { for some } X^{x, H} \text { as defined in (2.1) }\right\} .
$$

Our agent has a utility function $U: \mathbb{R}_{+} \rightarrow \mathbb{R}_{+}$satisfying,

\section{Assumption 2.1 (Generalized Inada Conditions)}

$$
\inf \bigcup_{x \in \mathbb{R}_{+}} \partial U(x)=0, \quad \sup \bigcup_{x \in \mathbb{R}_{+}} \partial U(x)=\infty
$$

Throughout the paper $\partial U$ denotes the subdifferential of the function $U$. In addition we require 


\section{Assumption 2.2}

$$
U(0)=0, U(\infty)=\infty \text { and } U \text { is concave and increasing on } \mathbb{R}_{+} .
$$

Remark 2.3 The assumption that $U(0)=0$ is not essential, however it aids comparison with [3], where the original statement of the conjecture appears. Our counterexample does in fact not depend on this assumption and one can derive similar phenomena for utility functions satisfying either $U(0) \geq-\infty$ or $U(\infty)<\infty$, or both.

We can now formulate the utility maximization problem,

$$
u(x):=\sup _{X \in \mathcal{X}(x)} \mathbb{E}[U(X)] .
$$

The standard solution technique is via an appropriate dual problem. We introduce the conjugate (or dual) function to $U$,

$$
\tilde{U}(y):=\sup _{x>0}\{U(x)-x y\} .
$$

Due to Assumption 2.2 this is a convex decreasing nonnegative function. From [8] it is known that to guarantee the existence of an optimal solution we must impose a condition on the asymptotic elasticity (AE) of the utility function $U$. In [3] the authors show that, for a nonsmooth utility function, this should be put on the dual function. Define

$$
\operatorname{AE}(\tilde{U}):=\limsup _{y \rightarrow 0} \sup _{q \in \partial \tilde{U}(y)} \frac{|q| y}{\tilde{U}(y)} .
$$

Assumption 2.4 $\mathrm{AE}(\tilde{U})<\infty$.

For $y>0$ we define the set of dual variables

$$
\mathcal{Y}(y):=\left\{Y \in L_{+}^{0}(\mathbb{P}): \mathbb{E}[X Y] \leq x y \text { for all } X \in \mathcal{X}(x)\right\} .
$$

We write $\mathcal{M}$ for the set of equivalent local martingale measures for $S$ and require the following assumption of no arbitrage type,

Assumption 2.5 $\mathcal{M} \neq \emptyset$.

The corresponding dual problem is given by

$$
w(x):=\inf _{y>0} \inf _{Y \in \mathcal{Y}(y)}(\mathbb{E}[\tilde{U}(Y)]+x y) .
$$

We now recall [2] Theorem 3.2.

Theorem 2.6 Suppose that Assumptions 2.1 2.2, 2.4 and 2.5 hold. Let $x>0$ be such that $w(x)<\infty$. Then

(i) There exist $y_{*}>0$ and $Y_{*} \in \mathcal{Y}\left(y_{*}\right)$ optimal for the dual problem, i.e.,

$$
w(x)=\mathbb{E}\left[\tilde{U}\left(Y_{*}\right)+x y_{*}\right] .
$$


(ii) There exists $X_{*} \in \mathcal{X}(x)$ optimal for the primal problem such that,

$$
X_{*} \in-\partial \tilde{U}\left(Y_{*}\right) \text { and } \mathbb{E}\left[X_{*} Y_{*}\right]=x y_{*} .
$$

(iii) There is no duality gap, i.e.,

$$
u(x)=w(x)=\inf _{y>0} \inf _{\mathbb{Q} \in \mathcal{M}} \mathbb{E}\left[\tilde{U}\left(y \frac{d \mathbb{Q}}{d \mathbb{P}}\right)+x y\right] .
$$

The last equality follows from [2] Remark 3.9.

Let us now examine Theorem 2.6 (ii) a little closer. Rockafellar [10] Theorem 23.5. states that, for conjugate functions $U$ and $\tilde{U}$,

$$
\begin{gathered}
U(x) \leq \tilde{U}(y)+x y \text { for all } y \geq 0, \\
U(x)=\tilde{U}(y)+x y \text { if and only if } x \in-\partial \tilde{U}(y) .
\end{gathered}
$$

Suppose that $\left(y_{*}, Y_{*}\right)$ are optimal for the dual problem and that we may choose $\hat{X} \in-\partial \tilde{U}\left(Y_{*}\right)$ satisfying $\mathbb{E}\left[\hat{X} Y_{*}\right]=x y_{*}$. It then follows that

$$
\mathbb{E}[U(\hat{X})]=\mathbb{E}\left[\tilde{U}\left(Y_{*}\right)+\hat{X} Y_{*}\right]=\mathbb{E}\left[\tilde{U}\left(Y_{*}\right)\right]+x y_{*} \geq \sup _{X \in \mathcal{X}(x)} \mathbb{E}[U(X)] .
$$

It is now apparent that $\hat{X}$ is optimal if and only if it is an element of $\mathcal{X}(x)$. The following conjecture, [3] Remark 4.6, is suggested by D.Ocone,

Conjecture 2.7 All random variables $\hat{X}$ valued in $-\partial \tilde{U}\left(Y_{*}\right)$ for which $\mathbb{E}\left[\hat{X} Y_{*}\right]=$ $x y_{*}$ satisfy

$$
\hat{X} \in \mathcal{X}(x)
$$

so that $u(x)=\mathbb{E}[U(\hat{X})]$.

Remark 2.8 We note that, strictly speaking, this is not how the authors formulate the conjecture in [3]. Instead they simply conjecture that every random variable valued in $-\partial \tilde{U}\left(Y_{*}\right)$ is an element of $\mathcal{X}(x)$ and is therefore optimal. However it is clear that they actually intend the above and so we only discuss this here.

Let us first observe that the set of random variables satisfying the conditions of Conjecture 2.7 is nonempty as the $X_{*}$ from Theorem 2.6 (ii) has these properties. In order for this conjecture to be interesting and merit some study it is necessary to exclude some trivial cases and we isolate them here. When the utility function is strictly concave its dual is differentiable and the relevant subdifferential is a singleton, moreover we have that the primal solution is unique. This means that the conjecture is immediately true. It is thus necessary to insist that $U$ be concave but not strictly concave. By this we mean that it is concave but has some linear section.

On the other hand, if the market is complete, then the dual minimizer is simply a constant, precisely $y_{*}$, multiplied by the Radon-Nikodym derivative of the unique martingale measure. It then follows that replicability is equivalent to the budget constraint occurring in Conjecture 2.7 so that the answer is trivially positive. 


\section{Counterexample}

\subsection{Model and Optimal Solutions}

We begin by introducing the utility function $U$,

$$
U(x):= \begin{cases}2 \sqrt{x} & x \in[0,1] \\ x+1 & x \in(1,5) \\ 2 \sqrt{x-4}+4 & x \in[5, \infty) .\end{cases}
$$

We take a power utility function and adapt it to ensure that it is not strictly concave. One can verify that $U$ is continuously differentiable and satisfies Assumptions 2.1 and 2.2. We may compute the dual of $U$ and this is given by

$$
\tilde{U}(y)= \begin{cases}4-4 y+\frac{1}{y} & y \in(0,1) \\ \frac{1}{y} & y \in[1, \infty) .\end{cases}
$$

Since $\tilde{U}$ is differentiable for $|y|<1$ a calculation shows that Assumption 2.4 is satisfied. Crucial in our analysis is the fact that $\tilde{U}$ is not continuously differentiable. Indeed, we have the following

$$
\partial \tilde{U}(y)= \begin{cases}-4-\frac{1}{y^{2}} & y \in(0,1) \\ {[-5,-1]} & y=1 \\ -\frac{1}{y^{2}} & y \in(1, \infty) .\end{cases}
$$

Next we describe our market. Let $\left(\Omega, \mathcal{F},\left(\mathcal{G}_{t}\right)_{t \geq 0}, \mathbb{P}\right)$ be a filtered probability space on which a process $W$ and a random variable $\xi$ are defined.

(i) The process $W$ is a standard Brownian motion and $\left(\mathcal{G}_{t}\right)_{t \geq 0}$ is the augmented filtration generated by $W$.

(ii) The random variable $\xi$ is independent of the entire path of $W$ and valued in $\{0,1\}$ with the following distribution,

$$
\mathbb{P}(\xi=0)=\frac{1}{3}, \quad \mathbb{P}(\xi=1)=\frac{2}{3} .
$$

To construct the filtration for our example we take $\left(\mathcal{G}_{t}\right)_{t \geq 0}$ and define

$$
\mathcal{F}_{t}:=\sigma\left(\mathcal{G}_{t}, \sigma(\xi)\right)
$$

That is to say we augment the natural filtration of Brownian motion to ensure that it satisfies the usual conditions and that the random variable $\xi$ is always measurable. Henceforth we consider $\left(\Omega, \mathcal{F},\left(\mathcal{F}_{t}\right)_{t \geq 0}, \mathbb{P}\right)$.

We introduce the stopping time $\tau$, defined by

$$
\tau:=\inf \left\{t>0: W_{t}+\frac{t}{2} \notin(-\log 4, \log 4)\right\},
$$

then set the terminal time horizon for utility maximization to be

$$
T:=\tau \text {. }
$$


A calculation based on the first exit time of a Brownian motion with drift, see Rogers and Williams [11] shows that

$$
\mathbb{P}\left(W_{\tau}+\frac{\tau}{2}=\log 4\right)=\frac{4}{5}, \quad \mathbb{P}\left(W_{\tau}+\frac{\tau}{2}=-\log 4\right)=\frac{1}{5} .
$$

Our asset $S$ is then defined by

$$
S_{t}:=\mathbf{1}_{\{\xi=0\}}+\exp \left(W_{t \wedge \tau}+\frac{1}{2}(t \wedge \tau)\right) \mathbf{1}_{\{\xi=1\}} .
$$

We observe that $S$ has continuous paths, is uniformly bounded, nonnegative and at time $T$ is valued in the set $\{1 / 4,1,4\}$. We may describe $S$ as follows, at time 0 we flip a biased coin, with the probability of heads equal to $\frac{2}{3}$, modelled by the random variable $\xi$. If we get a tail we do nothing $(\xi=0)$ whereas in the case of a head we run a submartingale formed from the Brownian motion until it hits some predefined level.

The purpose of the random variable $\xi$ is to ensure the market is incomplete and is inspired by Schachermayer $[12,13]$. The crucial point is that there is a unique equivalent martingale measure for the process

$$
V_{t}:=\exp \left(W_{t \wedge \tau}+\frac{1}{2}(t \wedge \tau)\right)
$$

This means that we may construct a large family of martingale measures simply by changing the distribution of the random variable $\xi$. This is equivalent to determining the pair $\left(q_{0}, q_{1}\right)$ where

$$
\mathbb{Q}(\xi=0)=q_{0}, \quad \mathbb{Q}(\xi=1)=q_{1} .
$$

For consistency we set $p_{0}=\frac{1}{3}$ and $p_{1}=\frac{2}{3}$. Let $q=\left(q_{0}, q_{1}\right)$ and consider processes $Z^{q}$, defined for $t \geq 0$ by

$$
Z_{t}^{q}:=\frac{q_{0}}{p_{0}} \mathbf{1}_{\{\xi=0\}}+S_{t}^{-1} \frac{q_{1}}{p_{1}} \mathbf{1}_{\{\xi=1\}} .
$$

Using the independence of $W$ and $\xi$ one can verify that $Z^{q}$ is a $\mathbb{P}$-martingale for $q_{0}, q_{1}>0$ such that $q_{0}+q_{1}=1$. Moreover, for any such $q, Z^{q} S$ is a $\mathbb{P}$-martingale (it is constant). This implies that if we define

$$
\frac{d \mathbb{Q}^{q}}{d \mathbb{P}}=Z_{T}^{q},
$$

then $\mathbb{Q}^{q} \in \mathcal{M}$ and it follows that the market is incomplete. Recalling the comments following Conjecture 2.7 we observe that our utility function is not strictly concave and our market is incomplete so that the conjecture is nontrivial.

Our idea is the following, we first look at the utility maximization problem for constant investment strategies. We then apply duality techniques to show that this local maximizer is in fact global. Having relatively simple forms for the optimal terminal wealth and a set of equivalent martingale measures then allows us to construct a random variable with the required pathological properties. 
We focus on $u(1)$ and consider

$$
\begin{aligned}
\mathcal{X}^{\text {const }}(1) & =\left\{X \in L_{+}^{0}(\mathbb{P}): X \leq 1+H\left(S_{T}-S_{0}\right), \text { for } H \in \mathbb{R}\right\} \\
& =\left\{X \in L_{+}^{0}(\mathbb{P}): X \leq 1+H\left(S_{T}-S_{0}\right), \text { for } H \in\left[-\frac{1}{3}, \frac{4}{3}\right]\right\} .
\end{aligned}
$$

The second representation follows from the nonnegative admissibility requirement. Define the maximization problem

$$
u^{\text {const }}(1):=\sup _{X \in \mathcal{X}^{\text {const }}(1)} \mathbb{E}[U(X)] .
$$

In the present setting we may rewrite this as follows,

$$
u^{\mathrm{const}}(1)=\sup _{H \in\left[-\frac{1}{3}, \frac{4}{3}\right]}\left[\frac{8}{15} U(1+3 H)+\frac{2}{15} U\left(1-\frac{3}{4} H\right)+\frac{1}{3} U(1)\right] .
$$

Since $U$ is continuously differentiable, one maximizes in the usual fashion to obtain $H_{*}=\frac{85}{64}$ as the optimal replicating strategy. We define $X_{*}:=1+\left(H_{*}\right.$. $S)_{T}$ and a calculation gives

$$
X_{*}=\mathbf{1}_{\{\xi=0\}}+\frac{319}{64} \mathbf{1}_{\left\{S_{T}=4, \xi=1\right\}}+\frac{1}{256} \mathbf{1}_{\left\{S_{T}=\frac{1}{4}, \xi=1\right\}} .
$$

Observe that $X_{*}$ is constant on the above 3 sets which partition $\Omega$, this allows us to write it as a simple function. The next step is to show that in fact $X_{*}$ is the optimal terminal wealth when non constant strategies are allowed. We evaluate $U^{\prime}\left(X_{*}\right)$, a random variable valued in $\{1,16\}$,

$$
U^{\prime}\left(X_{*}\right)=\mathbf{1}_{\{\xi=0\}}+\mathbf{1}_{\left\{S_{T}=4, \xi=1\right\}}+16 \mathbf{1}_{\left\{S_{T}=\frac{1}{4}, \xi=1\right\}} .
$$

We may equivalently write this as

$$
U^{\prime}\left(X_{*}\right)=3\left(\frac{q_{0}^{*}}{p_{0}} \mathbf{1}_{\{\xi=0\}}+S_{T}^{-1} \frac{q_{1}^{*}}{p_{1}} \mathbf{1}_{\{\xi=1\}}\right),
$$

where $q_{0}^{*}=\frac{1}{9}$ and $q_{1}^{*}=\frac{8}{9}$. We compare this with (3.2) and write $\mathbb{Q}_{*}$ for the risk neutral measure having Radon-Nikodym derivative $Z_{T}^{q^{*}}$. It follows that,

$$
Y_{*}:=U^{\prime}\left(X_{*}\right)=3 \frac{d \mathbb{Q}_{*}}{d \mathbb{P}} \in \mathcal{Y}(3)
$$

A calculation shows that

$$
\mathbb{E}\left[X_{*} Y_{*}\right]=3 .
$$

If we set $y_{*}:=3$ then via the conjugacy relations we have

$$
\mathbb{E}\left[\tilde{U}\left(Y_{*}\right)\right]+3=\mathbb{E}\left[\tilde{U}\left(y_{*} \frac{d \mathbb{Q}_{*}}{d \mathbb{P}}\right)+y_{*} X_{*} \frac{d \mathbb{Q}_{*}}{d \mathbb{P}}\right]=\mathbb{E}\left[U\left(X_{*}\right)\right] .
$$

Combining this with the inequality

$$
\mathbb{E}\left[U\left(X_{*}\right)\right] \leq u(1) \leq \inf _{y>0} \inf _{\mathbb{Q} \in \mathcal{M}} \mathbb{E}\left[\tilde{U}\left(y \frac{d \mathbb{Q}}{d \mathbb{P}}\right)+y\right]
$$


It follows that optimal solutions are

$$
H_{*}=\frac{85}{64}, \quad X_{*}=\left\{\begin{array}{ll}
\frac{319}{64} & S_{T}=4 \\
1 & S_{T}=1 \\
\frac{1}{256} & S_{T}=\frac{1}{4}
\end{array} \quad\left(y_{*}, Y_{*}\right)=\left(3,3 \frac{d \mathbb{Q}_{*}}{d \mathbb{P}}\right) .\right.
$$

Remark 3.1 We note that due to the fact that $U$ is not strictly concave we may not have uniqueness.

\subsection{The Subgradient Valued Random Variable}

Having found optimal solutions our goal is to construct a random variable $\hat{X} \in-\partial \tilde{U}\left(Y_{*}\right)$, satisfying $\mathbb{E}\left[\hat{X} Y_{*}\right]=y_{*}$, that is not an element of $\mathcal{X}(1)$ (recall that $x=1$ in the present discussion). Using (3.1),

$$
-\partial \tilde{U}\left(Y_{*}\right)= \begin{cases}{[1,5]} & Y_{*}=1 \\ \frac{1}{256} & Y_{*}=16 .\end{cases}
$$

We define the following random variable,

$$
\hat{X}:=\frac{359}{104} \mathbf{1}_{\{\xi=0\}}+\frac{359}{104} \mathbf{1}_{\left\{S_{T}=4, \xi=1\right\}}+\frac{1}{256} \mathbf{1}_{\left\{S_{T}=\frac{1}{4}, \xi=1\right\}} .
$$

Comparing this with (3.3) and (3.6) shows that indeed $\hat{X} \in-\partial \tilde{U}\left(Y_{*}\right)$. A calculation then shows that $\mathbb{E}\left[\hat{X} Y_{*}\right]=3=x y_{*}$. Our claim is therefore that $\hat{X} \notin \mathcal{X}(1)$. For a nonnegative random variable it follows from Delbaen and Schachermayer $[4,5]$ that

$$
\hat{X} \in \mathcal{X}(1) \text { if and only if } \sup _{\mathbb{Q} \in \mathcal{M}} \mathbb{E}_{\mathbb{Q}}[\hat{X}] \leq 1 .
$$

In particular we need only find $\hat{q}$ such that $\mathbb{E}\left[\hat{X} Z_{T}^{\hat{q}}\right]>1$ to establish the result. Since $\hat{X}>0$,

$$
\mathbb{E}\left[\hat{X} Z_{T}^{q}\right]>\mathbb{E}\left[\hat{X} Z_{T}^{q} \mathbf{1}_{\{\xi=0\}}\right]=\frac{359}{104} q_{0} .
$$

If we choose $\hat{q}_{0}=\frac{1}{2}$ and $\hat{q}_{1}=\frac{1}{2}$ then,

$$
\mathbb{E}\left[\hat{X} Z_{T}^{\hat{q}}\right]>\frac{359}{208}>1 .
$$

Thus $\hat{X}$ is not an element of $\mathcal{X}(1)$ and we have constructed a counterexample to Conjecture 2.7.

We conclude this section by discussing the construction of $\hat{X}$, in particular its relationship to the left and right derivatives of $\tilde{U}$. Recall that $\tilde{U}$ is a convex decreasing function, it then follows that for $y \geq 0$,

$$
\partial \tilde{U}(y)=\left[D^{-} \tilde{U}(y), D^{+} \tilde{U}(y)\right],
$$


where $D^{-} \tilde{U}$ and $D^{+} \tilde{U}$ denote the left and right derivatives respectively. This gives a representation for the subdifferential as a random interval.

$$
-\partial \tilde{U}\left(Y_{*}\right)=\left[-D^{+} \tilde{U}\left(Y_{*}\right),-D^{-} \tilde{U}\left(Y_{*}\right)\right] .
$$

We are interested in the relation between $\hat{X}$ and the random variables $X^{-}:=$ $-D^{+} \tilde{U}\left(Y_{*}\right)$ and $X^{+}:=-D^{-} \tilde{U}\left(Y_{*}\right)$. Select an arbitrary $Y \in \mathcal{Y}(y)$ and for $0<$ $\varepsilon<1$ form the random variable $Y_{\varepsilon}:=Y_{*}+\varepsilon Y$ together with the constant $y_{\varepsilon}:=$ $y_{*}+\varepsilon y$. Let us slightly abuse notation and write the subgradient inequality as

$$
\tilde{U}(z) \geq \tilde{U}(y)+\partial \tilde{U}(y)(z-y) .
$$

Since $Y_{\varepsilon} \in \mathcal{Y}\left(y_{\varepsilon}\right)$ and the pair $\left(y_{*}, Y_{*}\right)$ is the dual minimizer we have

$$
\begin{aligned}
0 & \geq \frac{1}{\varepsilon}\left(\mathbb{E}\left[\tilde{U}\left(Y_{*}\right)-\tilde{U}\left(Y_{\varepsilon}\right)\right]+x\left(y_{*}-y_{\varepsilon}\right)\right) \\
& \geq \mathbb{E}\left[-D^{+} \tilde{U}\left(Y_{\varepsilon}\right) Y\right]-x y
\end{aligned}
$$

where the second inequality follows from (3.8) by choosing $-D^{+} \tilde{U}\left(Y_{\varepsilon}\right) \in$ $\partial \tilde{U}\left(Y_{\varepsilon}\right)$. Proceeding similarly to [8] Lemma 3.9 and using the right continuity of $-D^{+} \tilde{U}$, one can show that (3.9) implies $X^{-} \in \mathcal{X}(x)$, in particular $\mathbb{E}\left[X^{-} Y_{*}\right] \leq x y_{*}$.

If instead one defines $Y_{\varepsilon}:=(1-\varepsilon) Y_{*}$ and $y_{\varepsilon}:=(1-\varepsilon) y_{*}$ then proceeding analogously one can deduce the inequality

$$
\mathbb{E}\left[X^{+} Y_{*}\right] \geq x y_{*} .
$$

The random variables $X^{-}$and $X^{+}$are subdifferential valued and a naive candidate optimal solution would be formed by taking a suitable convex combination to ensure the budget constraint with equality were satisfied. In our case we have

$$
X^{-}:=\mathbf{1}_{\{\xi=0\}}+\mathbf{1}_{\left\{S_{T}=4, \xi=1\right\}}+\frac{1}{256} \mathbf{1}_{\left\{S_{T}=\frac{1}{4}, \xi=1\right\}}
$$

and

$$
X^{+}:=5 \mathbf{1}_{\{\xi=0\}}+5 \mathbf{1}_{\left\{S_{T}=4, \xi=1\right\}}+\frac{1}{256} \mathbf{1}_{\left\{S_{T}=\frac{1}{4}, \xi=1\right\}} .
$$

We look at convex combinations, $X^{\lambda}:=\lambda X^{-}+(1-\lambda) X^{+}$for $\lambda \in[0,1]$ and choose $\lambda^{*}$ such that $\mathbb{E}\left[X^{\lambda_{*}} Y_{*}\right]=x y_{*}(=3)$. A calculation shows that $\lambda_{*}=\frac{161}{414}$. This is precisely the method used to construct the $\hat{X}$ appearing in (3.7), i.e. $\hat{X}=X^{\lambda_{*}}$. The reason we construct $\hat{X}$ this way is that in addition to providing a counterexample to Conjecture 2.7 we can establish an interesting related result. Specifically, in the general case the optimal solution for the primal problem cannot be written as a deterministic linear combination of the left and right derivatives of the dual function evaluated at the dual minimizer. 
Remark 3.2 In the original setting of the conjecture the authors assume that the initial filtration is trivial. Looking at our setting above this is not the case, indeed the initial sigma algebra contains the knowledge of the random variable $\xi$. A simple remedy to this is as follows, one starts with a trivial sigma algebra and holds everything constant for a deterministic period of time, say 1 . At $t=1$ flip the coin and construct a "time shifted" $S$. The new model created in this way satisfies exactly the conditions of [3]. For ease of presentation we do not include the described modification.

Remark 3.3 We could equivalently formulate a discrete time one period analog of our example. There are two reasons why we chose to give the present continuous time version. Firstly in [3], where the conjecture was orignally formulated, their market model is a continuous semimartingale $S$. Secondly our counterexample shows that enforcing more regularity on the paths of $S$ does not improve the situation and one can still observe the same phenomena.

\section{A Generalization of the Conjecture}

Let us again consider the Theorem 2.6. Using the notation therein, any random variable $\hat{X}$ satisfying all of

$$
\text { (I) } \hat{X} \in \mathcal{X}(x), \quad(\text { II }) \hat{X} \in-\partial \tilde{U}\left(Y_{*}\right), \quad \text { (III) } \mathbb{E}\left[\hat{X} Y_{*}\right]=x y_{*}
$$

is optimal. From the present viewpoint Conjecture 2.7 simply asks whether (II) and (III) automatically imply (I)? It therefore seems natural to investigate whether there are any other dependence relations between (I), (II) and (III).

Lemma 4.1 In the example constructed in Section 3 no two of (I), (II) and (III) are sufficient to imply the third.

Proof We have already shown that (II) and (III) do not imply (I).

In the model of Section 3, define the constant $\hat{H}:=\frac{4}{3}$, together with the random variable $\hat{X}:=1+(\hat{H} \cdot S)_{T}$, an element in $\mathcal{X}(1)$. A calculation shows that

$$
\hat{X}=\mathbf{1}_{\{\xi=0\}}+5 \mathbf{1}_{\left\{S_{T}=4, \xi=1\right\}}+0 \mathbf{1}_{\left\{S_{T}=\frac{1}{4}, \xi=1\right\}} .
$$

In particular we have

$$
\mathbb{E}\left[\hat{X} Y_{*}\right]=3=x y_{*} .
$$

However $\hat{X} \notin-\partial \tilde{U}\left(Y_{*}\right)$ a.s. as $Y_{*}$ is bounded and $0 \in-\partial \tilde{U}(y)$ if and only if $y=\infty$. This shows that (I) and (III) do not imply (II).

For the final interdependence consider the random variable,

$$
\hat{X}:=\mathbf{1}_{\{\xi=0\}}+\mathbf{1}_{\left\{S_{T}=4, \xi=1\right\}}+\frac{1}{256} \mathbf{1}_{\left\{S_{T}=\frac{1}{4}, \xi=1\right\}} .
$$

In fact, $\hat{X}=X^{-}$, where $X^{-}$is defined in (3.10) and it therefore follows that $\hat{X} \in-\partial \tilde{U}\left(Y_{*}\right)$. Moreover since $\hat{X} \leq X_{*}$ we have $\hat{X} \in \mathcal{X}(1)$. However a calculation shows that

$$
\mathbb{E}\left[\hat{X} Y_{*}\right]=\frac{7}{8}<3=x y_{*} .
$$

Thus (I) and (II) do not imply (III). 
We finish with a corollary which gives the necessary and sufficient conditions for optimality in the primal problem.

Corollary 4.2 Suppose that $U$ is a concave increasing function defined on $\mathbb{R}_{+}$such that Assumptions 2.1, 2.4 and 2.5 hold.

Let $y_{*}>0, Y_{*} \in \mathcal{Y}\left(y_{*}\right)$ be the solution to the dual problem and $x>0$ such that $w(x)<\infty$. The random variable $\hat{X}$ is optimal for the primal problem if and only if

$$
\text { (I) } \hat{X} \in \mathcal{X}(x), \quad(I I) \hat{X} \in-\partial \tilde{U}\left(Y_{*}\right), \quad \text { (III) } \mathbb{E}\left[\hat{X} Y_{*}\right]=x y_{*} .
$$

Remark 4.3 When $U$ is strictly concave it is shown in [8] that (II) implies both (I) and (III). On the other hand when $U$ is not strictly concave Lemma 4.1 shows that (I), (II) and (III) are the minimum sufficient conditions for optimality.

Acknowledgements We thank Jan Obłój for some helpful discussions as well as two anonymous referees whose comments helped improve a previous version of this paper.

\section{References}

1. B. Bouchard. Utility maximization on the real line under proportional transaction costs. Finance Stoch., 6(4):495-516, 2002.

2. B. Bouchard, N. Touzi, and A. Zeghal. Dual formulation of the utility maximization problem: the case of nonsmooth utility. Ann. Appl. Probab., 14(2):678-717, 2004.

3. G. Deelstra, H. Pham, and N. Touzi. Dual formulation of the utility maximization problem under transaction costs. Ann. Appl. Probab., 11(4):1353-1383, 2001.

4. F. Delbaen and W. Schachermayer. A general version of the fundamental theorem of asset pricing. Math. Ann., 300(3):463-520, 1994.

5. F. Delbaen and W. Schachermayer. The fundamental theorem of asset pricing for unbounded stochastic processes. Math. Ann., 312(2):215-250, 1998.

6. J. Jacod and A. N. Shiryaev. Limit theorems for stochastic processes, volume 288 of Grundlehren der Mathematischen Wissenschaften [Fundamental Principles of Mathematical Sciences]. Springer-Verlag, Berlin, second edition, 2003.

7. I. Karatzas and G. Žitković. Optimal consumption from investment and random endowment in incomplete semimartingale markets. Ann. Probab., 31(4):1821-1858, 2003.

8. D. Kramkov and W. Schachermayer. The asymptotic elasticity of utility functions and optimal investment in incomplete markets. Ann. Appl. Probab., 9(3):904-950, 1999.

9. P. E. Protter. Stochastic integration and differential equations, volume 21 of Applications of Mathematics (New York). Springer-Verlag, Berlin, second edition, 2004. Stochastic Modelling and Applied Probability.

10. R. T. Rockafellar. Convex analysis. Princeton Mathematical Series, No. 28. Princeton University Press, Princeton, N.J., 1970.

11. L. C. G. Rogers and D. Williams. Diffusions, Markov processes, and martingales. Vol. 1. Cambridge Mathematical Library. Cambridge University Press, Cambridge, 2000. Foundations, Reprint of the second (1994) edition.

12. W. Schachermayer. How potential investments may change the optimal portfolio for the exponential utility. Working Paper, 2002.

13. W. Schachermayer. A super-martingale property of the optimal portfolio process. $F i$ nance Stoch., 7(4):433-456, 2003. 